TO APPEAR IN BEHAVIORAL ECOLOGY

\title{
Re-analyses of Shimoda et al. (2018): conception risk affects in- and extra-pair desire similarly
}

\author{
Talia N. Shirazi ${ }^{1}$, Benedict C. Jones ${ }^{2}$, James R. Roney ${ }^{3}$, Lisa DeBruine ${ }^{2}, \&$ David A. \\ Puts $^{1}$ \\ ${ }^{1}$ Department of Anthropology, Pennsylvania State University ${ }^{2}$ Institute of Neuroscience \& Psychology, \\ University of Glasgow ${ }^{3}$ Department of Psychological and Brain Sciences
}

Although sexual behavior is not under strict hormonal regulation across primates, a large body of work suggests robust ovulatory cycle shifts in women's sexual psychology and behavior. The adaptive value of such shifts, and the factors that modulate them, are actively debated. Do ovulatory cycle shifts in general libido facultatively allocate resources towards reproduction near ovulation when the benefit-cost ratio of engaging in sexual behavior is high (the Motivational Priorities hypothesis; Roney \& Simmons, 2013)? Do within-woman shifts in relative levels of in-pair versus extra-pair sexual desire function to recruit good genes for offspring when conception risk is high (DualMating hypothesis; Pillsworth \& Haselton, 2006)? Or are any observed shifts simply 'spandrels,' or nonfunctional byproducts of between-woman relationships (Havlíček et al. 2015)?

Addressing such questions, Shimoda, Campbell, and Barton (2018) evaluated whether partnered women's in-pair and extra-pair sexual desire change as a function of ovulatory cycle phase and whether such fluctuations are moderated by partner physical attractiveness or mutual relationship commitment. In 35 women in whom cycle phase was determined using luteinizing hormone (LH) tests and self-reports of menstrual onset, Shimoda et al. reported shifts in extra-pair $(p=0.01)$, but not in-pair $(p=0.07)$, sexual desire. The same general pattern of an increase in desire near ovulation was observed, however (Shimoda et al. 2018, Figure 1). Because shifts in sexual desire were not moderated by partner attractiveness or mutual commitment, Shimoda et al. concluded that their results support a byproduct account of women's ovulatory shifts. These findings contrast with recent work supporting the Motivational Priorities hypothesis, wherein both in-pair and extra-pair desire exhibit robust ovulatory shifts (see supplemental online materials for recommended reading).

However, there are several limitations in the analytical approach of Shimoda et al. Most critically, none of the analyses presented in Shimoda et al. directly compared the magnitude of putative shifts in in-pair versus extra-pair desire, even though doing so is crucial for discriminating among hypotheses (Gelman and Stern 2006). Concluding that the existence or magnitude of the shift in in-pair desire significantly differs from that of extra-pair desire requires a statistical model that simultaneously assesses both constructs, and directly tests for an interaction between desire type (i.e., in-pair and extra-pair desire) and conception risk. In addition, Shimoda et al. created a categorical cycle phase variable that collapses across days within each phase, despite the fact that 
conception risk varies more than four-fold within the peri-ovulatory phase. Hence, information about such within-phase changes was lost. Finally, fixed rather than random slopes were used due to model convergence problems; however, tests of multilevel models without random slopes can be prone to false positives.

Here, we address these limitations by reanalyzing Shimoda et al.'s open data (https://doi.org/10.5061/dryad.t5pr5.2) and obtain a different pattern of results. Harnessing the precision afforded by daily observations, we analyzed in-pair and extrapair desire as a function of daily conception risk. Ovulation-centered cycle day was assigned according to date of positive $\mathrm{LH}$ test, and nonzero conception risk estimates were assigned to days -5 (corresponding to 5 days prior to ovulation) through 0 using weighted estimates from prior studies (see supplementary online materials). In-pair and extra-pair desire were analyzed in the same model to assess whether putative relationships between desire and conception risk differed as a function of desire type. This model also assessed whether interactions between conception risk and moderators of interest, partner attractiveness and mutual commitment, differed as a function of desire type. Finally, we allowed for random slopes and specified them maximally. Our analyses were performed in R using the nlme4 package. All data and code files can be found at https://osf.io/r2vcz/.

In our model with mood entered as a time-varying covariate, there was a significant positive effect of conception risk on desire $(p=0.002)$, suggesting that overall levels of desire increase as conception risk increases (Figure 1). There was also a significant effect of desire type $(p<0.001)$, such that levels of in-pair desire exceeded levels of extra-pair desire. The positive effect of conception risk on desire was not moderated by interactions with partner attractiveness $(p=0.403)$, mutual commitment $(p=0.637)$, or importantly, desire type $(p=0.302)$, demonstrating that the association between conception risk and in-pair desire did not significantly differ from that of conception risk and extra-pair desire. The conception risk $\times$ partner attractiveness $\times$ desire type $(p=0.240)$ and conception risk $\times$ mutual commitment $\times$ desire type interactions $(p=0.843)$ were also nonsignificant. All patterns were robust to the exclusion of mood as a covariate. When analyzed separately, our analyses also showed a significant positive effect of conception risk on in-pair desire $(p=0.014)$ that was not significant for extra-pair desire $(p=0.086)$. We note that this is opposite to the results of Shimoda et al., but again, the effect of conception risk did not differ significantly across desire types in our full model. 


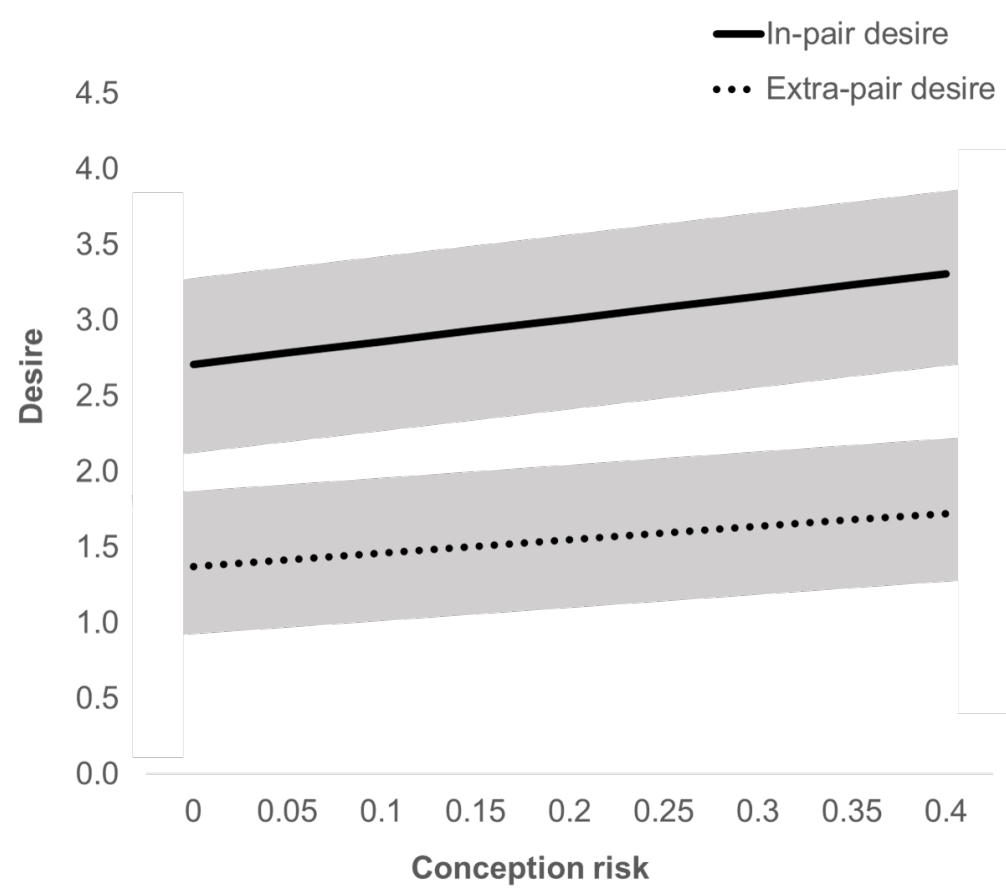

Figure 1. Estimated effect of conception risk on in-pair and extra-pair sexual desire. Grey shading indicates estimated beta standard errors.

These results are consistent with the Motivational Priorities hypothesis, the theoretical framework wherein increases in general sexual desire, regardless of target, channel resources towards cognition and behavior to facilitate reproduction when the chances of conception are greatest (Roney and Simmons 2013). Although the observed changes were modest in magnitude, we note that even small changes in phenotypes that are as closely tied to reproduction as sexual desire and behavior can have measurable and important fitness consequences. The cyclic shift in desire that Shimoda et al. argued was specific to the extra-pair context, actually appears to generalize across contexts. These results are thus not consistent with a byproduct account of women's ovulatory shifts in sexual psychology, as Shimoda et al. suggest, but rather with functional psychobehavioral shifts that may have evolved to increase reproductive success. That cyclic shifts in in-pair and extra-pair desire were not modulated by partner attractiveness or mutual commitment casts doubt upon hypotheses, such as the Dual-Mating hypothesis, that argue relationship-specific or partner-specific variables significantly predict cyclic shifts in patterns of sexual desire.

Shimoda et al. address important, contentious questions with methodological rigor: For which outcomes do ovulatory cycle shifts appear, what variables modulate these shifts, and what (if any) are their adaptive functions? However, improved treatment and analysis of their data suggests answers to these questions that differ in theoretically meaningful ways. 


\section{References}

Gelman A, Stern H. 2006. The difference between "significant" and "not significant" is not itself statistically significant. Am Stat. 60(4):328-331.

doi:10.1198/000313006X152649.

Havlíček J, Cobey KD, Barrett L, Klapilová K, Roberts SC. 2015. The spandrels of Santa Barbara? A new perspective on the peri-ovulation paradigm. Behav Ecol. 26(5):1249-1260. doi:10.1093/beheco/arv064.

Pillsworth EG, Haselton MG. 2006. Women's sexual strategies: The evolution of longterm bonds and extrapair sex. Annu Rev Sex Res. 17:59-100.

Roney JR, Simmons ZL. 2013. Hormonal predictors of sexual motivation in natural menstrual cycles. Horm Behav. 63(4):636-645. doi:10.1016/j.yhbeh.2013.02.013.

Shimoda R, Campbell A, Barton RA. 2017. Women's emotional and sexual attraction to men across the menstrual cycle. Behav Ecol. 00:1-9. doi:10.1093/beheco/arx124. 